\title{
Decay of Solutions of Wave-type Pseudo-differential Equations over $p$-adic Fields
}

By

\author{
W. A. Zuniga-Galindo*
}

\begin{abstract}
We show that the solutions of $p$-adic pseudo-differential equations of wave type have a decay similar to the solutions of classical generalized wave equations.
\end{abstract}

\section{$\S 1$. Introduction}

During the eighties several physical models using $p$-adic numbers were proposed. Particularly various models of $p$-adic quantum mechanics [11], [13], [21], [22]. As a consequence of this fact several new mathematical problems emerged, among them, the study of $p$-adic pseudo-differential equations [8], [22]. In this paper we initiate the study of the decay of the solutions of wave-type pseudodifferential equations over $p$-adic fields; these equations were introduced by Kochubei [9] in connection with the problem of characterizing the $p$-adic wave functions using pseudo-differential operators. We show that the solutions of $p$-adic wave-type equations have a decay similar to the solutions of classical generalized wave equations.

Let $K$ be a $p$-adic field, i.e. a finite extension of $\mathbb{Q}_{p}$. Let $R_{K}$ be the valuation ring of $K, P_{K}$ the maximal ideal of $R_{K}$, and $\bar{K}=R_{K} / P_{K}$ the residue field of $K$. Let $\pi$ denote a fixed local parameter of $R_{K}$. The cardinality

Communicated by Y. Takahashi. Received September 21, 2004. Revised November 24, 2004 .

2000 Mathematics Subject Classification(s): Primary, 35S99, 47S10; Secondary 11S40.

Keywords: Non-archimedean pseudo-differential equations, restriction of Fourier transforms, exponential sums modulo $p^{m}$, Igusa local zeta function.

Project sponsored by the National Security Agency under Grant Number H98230-06-10040. The United States Government is authorized to reproduce and distribute reprints notwithstanding any copyright notation herein.

* Department of Mathematics and Computer Science, Barry University, 11300 N.E. Second Avenue, Miami Shores, Florida 33161, USA.

e-mail: wzuniga@mail. barry.edu.

(C) 2006 Research Institute for Mathematical Sciences, Kyoto University. All rights reserved. 
of $\bar{K}$ is denoted by $q$. For $z \in K, v(z) \in \mathbb{Z} \cup\{+\infty\}$ denotes the valuation of $z$, and $|z|_{K}=q^{-v(z)}$. Let $\mathbb{S}\left(K^{n}\right)$ denote the $\mathbb{C}$-vector space of Schwartz-Bruhat functions over $K^{n}$, the dual space $\mathbb{S}^{\prime}\left(K^{n}\right)$ is the space of distributions over $K^{n}$. Let $\mathcal{F}$ denote the Fourier transform over $\mathbb{S}\left(K^{n+1}\right)$. The reader can consult any of the references [6], [22], [23] for an exposition of the theory of distributions over $p$-adic fields.

This article aims to study the following initial value problem:

$$
\left\{\begin{array}{l}
(H u)(x, t)=0, x \in K^{n}, t \in K \\
u(x, 0)=f_{0}(x),
\end{array}\right.
$$

where $n \geq 1, f_{0}(x) \in \mathbb{S}\left(K^{n}\right)$, and

$$
\begin{aligned}
H: \mathbb{S}\left(K^{n+1}\right) & \longrightarrow \\
\Phi & \longrightarrow \mathcal{F}_{(\tau, \xi) \longrightarrow(x, t)}^{-1}\left(|\tau-\phi(\xi)|_{K} \mathcal{F}_{(x, t) \longrightarrow(\tau, \xi)} \Phi\right),
\end{aligned}
$$

is a pseudo-differential operator with symbol $|\tau-\phi(\xi)|_{K}$, where $\phi(\xi)$ is a polynomial in $K\left[\xi_{1}, \ldots, \xi_{n}\right]$ satisfying $\phi(0)=0$. In the case in which $\phi(\xi)=$ $a_{1} \xi_{1}^{2}+\cdots+a_{n} \xi_{n}^{2}, H$ is called a Schrödinger-type pseudo-differential operator; this operator was introduced by Kochubei in [9]. For $n=1$ the solution of (1) appears in the formalism of $p$-adic quantum mechanics as the wave function for the free particle [21]. The problem of characterizing the $p$-adic wave functions as solutions of some pseudo-differential equation remains open.

Let $\Psi(\cdot)$ denote an additive character of $K$ trivial on $R_{K}$ but no on $P_{K}^{-1}$. By passing to the Fourier transform in (1) one gets that

$$
|\tau-\phi(\xi)|_{K} \mathcal{F}_{(x, t) \longrightarrow(\tau, \xi)} u=0 .
$$

Then any distribution of the form $\mathcal{F}^{-1} g$ with $g$ a distribution supported on $\tau-\phi(\xi)=0$ is a solution. By taking

$$
g(\xi, \tau)=\left(\mathcal{F}_{x \rightarrow \xi} f_{0}\right) \delta(\tau-\phi(\xi)),
$$

where $\delta$ is the Dirac distribution, one gets

$$
u(x, t)=\int_{K^{n}} \Psi\left(t \phi(\xi)+\sum_{i=1}^{n} x_{i} \xi_{i}\right)\left(\mathcal{F}_{x \rightarrow \xi} f_{0}\right)(\xi)|d \xi|,
$$

here $|d \xi|$ is the Haar measure of $K^{n}$ normalized so that $\operatorname{vol}\left(R_{K}^{n}\right)=1$.

In this paper we show that the decay of $u(x, t)$ is completely similar to the decay of the solution of the following initial value problem: 


$$
\left\{\begin{array}{l}
\frac{\partial u^{\operatorname{arch}}(x, t)}{\partial t}=i \phi(D) u^{\operatorname{arch}}(x, t), x \in \mathbb{R}^{n}, t \in \mathbb{R} \\
u^{\operatorname{arch}}(x, 0)=f_{0}(x),
\end{array}\right.
$$

here $\phi(D)$ is a pseudo-differential operator having symbol $\phi(\xi)$. In this case

$$
u^{\operatorname{arch}}(x, t)=\int_{\mathbb{R}^{n}} \exp 2 \pi i\left(t \phi(\xi)+\sum_{i=1}^{n} x_{i} \xi_{i}\right)\left(\mathcal{F}_{x \rightarrow \xi} f_{0}\right)(\xi) d \xi
$$

is the solution of the initial value problem $(3)$. If $\phi(\xi)=\xi_{1}^{2}+\cdots+\xi_{n}^{2}$, i.e. $\phi(D)$ is the Laplacian, $u^{\text {arch }}(x, t)$ satisfies

$$
\left\|u^{\operatorname{arch}}(x, t)\right\|_{L \frac{2(n+2)}{n}} \leq c\left\|f_{0}\right\|_{L^{2}},
$$

(see [19]). If $n=1$ and $\phi(\xi)=\xi^{3}, u^{\text {arch }}(x, t)$ satisfies

$$
\left\|u^{\operatorname{arch}}(x, t)\right\|_{L^{8}} \leq c\left\|f_{0}\right\|_{L^{2}},
$$

(see [10]). We show that $u(x, t)$ satisfies $(5)$, if $\phi(\xi)=\xi_{1}^{2}+\cdots+\xi_{n}^{2}$ (see Corollary 2), and that $u(x, t)$ satisfies $(6)$, if $\phi(\xi)=\xi^{3}$ (see Corollary 3). These two results are particular cases of our main result which describes the decay of $u(x, t)$ in $L^{\sigma}\left(K^{n+1}\right)$ when $\phi(\xi)$ is a non-degenerate polynomial with respect to its Newton polyhedron (see Theorem 6 ). The proof is achieved by adapting standard techniques in PDEs and by using number-theoretic techniques for estimating exponential sums modulo $\pi^{m}$. Indeed, like in the classical case the estimation of the decay rate can be reduced to the problem of estimating of the restriction of Fourier transforms to non-degenerate hypersurfaces [17]; we solve this problem (see Theorems 4,5 ) by reducing it to the estimation of exponential sums modulo $\pi^{m}$ (see Theorems 2,3). These exponential sums are related to the Igusa zeta function for non-degenerate polynomials [3], [7], [25], [26]. More precisely, by using Igusa's method, the estimation of these exponential sums can be reduced to the description of the poles of twisted local zeta functions [3], [25], [26]. It is important to mention that all the results of this paper are valid in positive characteristic, i.e. if $K=\mathbb{F}_{q}((T)), q=p^{n}$, and $p>c$. Here $c$ is a constant that depends on the Newton polyhedron of the polynomial $\phi$.

The restriction of Fourier transforms in $\mathbb{R}^{n}$ (see e.g. [17, Chapter VIII]) was first posed and partially solved by Stein [5]. This problem have been intensively studied during the last thirty years [1], [17], [19], [24]. Recently Mockenhaupt and Tao have studied the restriction problem in $\mathbb{F}_{q}^{n}[12]$. In this 
paper we initiate the study of the restriction problem in the non-archimedean field setting.

The author thanks to the referee for his/her careful reading of this paper.

\section{§2. The Non-archimedean Principle of the Stationary Phase}

Given $f(x) \in K[x], x=\left(x_{1}, \ldots, x_{m}\right)$, we denote by

$$
C_{f}(K)=\left\{z \in K^{m} \mid \frac{\partial f}{\partial x_{1}}(z)=\cdots=\frac{\partial f}{\partial x_{m}}(z)=0\right\}
$$

the critical set of the mapping $f: K^{m} \rightarrow K$. If $f(x) \in R_{K}[x]$, we denote by $\bar{f}(x)$ its reduction modulo $\pi$, i.e. the polynomial obtained by reducing the coefficients of $f(x)$ modulo $\pi$.

Give a compact open set $A \subset K^{m}$, we set

$$
E_{A}(z, f)=\int_{A} \Psi(z f(x))|d x|,
$$

for $z \in K$, where $|d x|$ is the normalized Haar measure of $K^{m}$. If $A=R_{K}^{m}$ we use the simplified notation $E(z, f)$ instead of $E_{A}(z, f)$. If $f(x) \in R_{K}[x]$, then

$$
E(z, f)=q^{-n m} \sum_{x \bmod \pi^{n}} \Psi(z f(x)) ;
$$

thus $E(z, f)$ is a generalized Gaussian sum.

Lemma 1. Let $f(x) \in R_{K}[x], x=\left(x_{1}, \ldots, x_{m}\right)$, be a non-constant polynomial. Let $A$ be the preimage of $\bar{A} \subseteq \mathbb{F}_{q}^{m}$ under the canonical homomorphism $R_{K}^{m} \rightarrow\left(R_{K} / P_{K}\right)^{m}$. If $C_{f}(K) \cap A=\emptyset$, then there exists a constant $I(f, A)$ such that

$$
E(z, f)=0, \text { for }|z|_{K}>q^{2 I(f, A)+1}
$$

Proof. We define

$$
I(f, a)=\min _{1 \leq i \leq m}\left\{v\left(\frac{\partial f}{\partial x_{i}}(a)\right)\right\},
$$

for any $a \in A$, and

$$
I(f, A)=\sup _{a \in A}\{I(f, a)\} .
$$

Since $A$ is compact and $C_{f}(K) \cap A=\emptyset, I(f, A)<\infty$. 
We denote by $a^{*}$ an equivalence class of $R_{K}^{m}$ modulo $\left(P_{K}^{I(f, A)+1}\right)^{m}$, and by $a \in R_{K}^{m}$ a fixed representative of $a^{*}$. By decomposing $A$ into equivalence classes modulo $\left(P_{K}^{I(f, A)+1}\right)^{m}$, one gets

$$
E(z, f)=\sum_{a^{*} \subseteq A} q^{-m(I(f, A)+1)} \int_{R_{K}^{m}} \Psi\left(z f\left(a+\pi^{I(f, A)+1} x\right)\right)|d x| .
$$

Thus, it is sufficient to show that $\int_{R_{K}^{m}} \Psi\left(z f\left(a+\pi^{I(f, A)+1} x\right)\right)|d x|=0$ for $|z|_{K}>q^{2 I(f, A)+1}$.

On the other hand, if $a=\left(a_{1}, \ldots, a_{m}\right)$, then

$$
\frac{f\left(a+\pi^{I(f, A)+1} x\right)-f(a)}{\pi^{I(f, A)+1+\alpha_{0}}}
$$

equals

$$
\sum_{i=1}^{m} \pi^{-\alpha_{0}} \frac{\partial f}{\partial x_{i}}(a)\left(x-a_{i}\right)+\pi^{I(f, A)+1-\alpha_{0}} \text { (higher order terms) }
$$

where

$$
\alpha_{0}=\min _{i}\left\{v\left(\frac{\partial f}{\partial x_{i}}(a)\right)\right\}
$$

Therefore

$$
f\left(a+\pi^{I(f, A)+1} x\right)-f(a)=\pi^{I(f, A)+1+\alpha_{0}} \widetilde{f}(x)
$$

with $\widetilde{f}(x) \in R_{K}[x]$, and since $C_{f}(K) \cap A=\emptyset$, there exists an $i_{0} \in\{1, \ldots, m\}$ such that

$$
\overline{\frac{\partial \widetilde{f}}{\partial x_{i_{0}}}}(\bar{a}) \neq 0 .
$$

We put $y=\Phi(x)=\left(\Phi_{1}(x), \ldots, \Phi_{m}(x)\right)$ where

$$
\Phi_{i}(x)=\left\{\begin{array}{cc}
\tilde{f}(x) & i=i_{0} \\
x_{i} & i \neq i_{0}
\end{array}\right.
$$

Since $\Phi_{1}(x), \ldots, \Phi_{m}(x)$ are restricted power series and

$$
\overline{J\left(\frac{\left(y_{1}, \ldots, y_{m}\right)}{\left(x_{1}, \ldots, x_{m}\right)}\right)}=\overline{\frac{\partial \widetilde{f}}{\partial x_{i_{0}}}}(\bar{a}) \neq 0,
$$


the non-archimedean implicit function theorem implies that $y=\Phi(x)$ gives a measure-preserving map from $R_{K}^{m}$ to $R_{K}^{m}$ (see [7, Lemma 7.43]). Therefore

$$
\begin{aligned}
& \int_{R_{K}^{m}} \Psi\left(z f\left(a+\pi^{I(f, A)+1} x\right)\right)|d x| \\
& \quad=\Psi(z f(a)) \int_{R_{K}} \Psi\left(z \pi^{I(f, A)+1+\alpha_{0}} y_{i_{0}}\right)\left|d y_{i_{0}}\right|=0,
\end{aligned}
$$

for $v(z)<-\left(I(f, A)+1+\alpha_{0}\right)$, i.e. for $|z|_{K}>q^{I(f, A)+1+\alpha_{0}}$, and a fortiori

$$
\int_{R_{K}^{m}} \Psi\left(z f\left(a+\pi^{I(f, A)+1} x\right)\right)|d x|=0,
$$

for $|z|_{K}>q^{2 I(f, A)+1}$ and any $a$.

Theorem 1. Let $f(x) \in K[x], x=\left(x_{1}, \ldots, x_{m}\right)$, be a non-constant polynomial. Let $B \subset K^{m}$ be a compact open set. If $C_{f}(K) \cap B=\emptyset$, then there exist a constant $c(f, B)$ such that

$$
E_{B}(z, f)=0, \text { for }|z|_{K} \geq c(f, B) .
$$

Proof. By taking a covering $\cup_{i}\left(y_{i}+\left(\pi^{\alpha} R_{K}\right)^{m}\right)$ of $B, E_{B}(z, f)$ can be expressed as linear combination of integrals of the form $E\left(z, f_{i}\right)$ with $f_{i}(x) \in$ $K[x]$. After changing $z$ by $z \pi^{\beta}$, we may suppose that $f_{i}(x) \in R_{K}[x]$. By applying Lemma 1 we get that $E\left(z, f_{i}\right)=0$, for $|z|_{K}>c_{i}$. Therefore

$$
E_{B}(z, f)=0, \text { for }|z|_{K}>\max _{i} c_{i} .
$$

We note that the previous result implies that

$$
E_{B}(z, f)=O\left(|z|_{K}^{-M}\right)
$$

for any $M \geq 0$. This is the standard form of the principle of the stationary phase.

\section{§3. Local Zeta Functions and Exponential Sums}

In this section we review some results about exponential sums and Newton polyhedra that will be used in the next section. For $x \in K$ we denote by $a c(x)=x \pi^{-v(x)}$ its angular component. Let $f(x) \in R_{K}[x], x=\left(x_{1}, \ldots, x_{m}\right)$ 
be a non-constant polynomial, and $\chi: R_{K}^{\times} \rightarrow \mathbb{C}^{\times}$a character of $R_{K}^{\times}$, the group of units of $R_{K}$. We formally put $\chi(0)=0$. To these data one associates the Igusa local zeta function,

$$
Z(s, f, \chi)=\int_{R_{K}^{n}} \chi(a c f(x))|f(x)|_{K}^{s}|d x|, s \in \mathbb{C},
$$

for $\operatorname{Re}(s)>0$, where $|d x|$ denotes the normalized Haar measure of $K^{n}$. The Igusa local zeta function admits a meromorphic continuation to the complex plane as a rational function of $q^{-s}$. Furthermore, it is related to the number of solutions of polynomial congruences modulo $\pi^{m}$ and exponential sums modulo $\pi^{m}[2],[7]$.

\section{§3.1. Exponential sums associated with non-degenerate polynomials}

We set $\mathbb{R}_{+}=\{x \in \mathbb{R} \mid x \geqq 0\}$. Let $f(x)=\sum_{l} a_{l} x^{l} \in K[x], x=$ $\left(x_{1}, \ldots, x_{m}\right)$ be a non-constant polynomial satisfying $f(0)=0$. The set $\operatorname{supp}(f)=\left\{l \in \mathbb{N}^{m} \mid a_{l} \neq 0\right\}$ is called the support of $f$. The Newton polyhedron $\Gamma(f)$ of $f$ is defined as the convex hull in $\mathbb{R}_{+}^{m}$ of the set

$$
\bigcup_{l \in \operatorname{supp}(f)}\left(l+\mathbb{R}_{+}^{m}\right) .
$$

We denote by $\langle\cdot, \cdot\rangle$ the usual inner product of $\mathbb{R}^{m}$, and identify $\mathbb{R}^{m}$ with its dual by means of it. We set

$$
\left\langle a_{\gamma}, x\right\rangle=m\left(a_{\gamma}\right)
$$

for the equation of the supporting hyperplane of a facet $\gamma$ (i.e. a face of codimension 1 of $\Gamma(f))$ with perpendicular vector $a_{\gamma}=\left(a_{1}, \ldots, a_{n}\right) \in \mathbb{N}^{n} \backslash\{0\}$, and $\sigma\left(a_{\gamma}\right):=\sum_{i} a_{i}$.

Definition 1. A polynomial $f(x) \in K[x]$ is called non-degenerate with respect to its Newton polyhedron $\Gamma(f)$, if it satisfies the following two properties: (i) $C_{f}(K)=\{0\} \subset K^{n}$; (ii) for every proper face $\gamma \subset \Gamma(f)$, the critical set $C_{f_{\gamma}}(K)$ of $f_{\gamma}(x):=\sum_{i \in \gamma} a_{i} x^{i}$ satisfies $C_{f_{\gamma}}(K) \cap(K \backslash\{0\})^{m}=\emptyset$.

We note that the above definition is not standard because it requires that the origin be an isolated critical point (see e.g. [3], [4], [26]). The condition (ii) can be replaced by

$$
\left\{x \in K^{m} \mid f_{\gamma}(x)=0\right\} \cap C_{f_{\gamma}}(K) \cap(K \backslash\{0\})^{m}=\emptyset .
$$


If $K$ has characteristic $p>0$, by using Euler's identity, it can be verified that condition (ii) in the above definition is equivalent to (10), if $p$ does not divide the $m\left(a_{\gamma}\right) \neq 0$, for any facet $\gamma$.

In [26] the author showed that if $f$ is non-degenerate with respect $\Gamma(f)$, then the poles of $\left(1-q^{-1-s}\right) Z\left(s, f, \chi_{\text {triv }}\right)$ and $Z(s, f, \chi), \chi \neq \chi_{\text {triv }}$, have the form

$$
s=-\frac{\sigma\left(a_{\gamma}\right)}{m\left(a_{\gamma}\right)}+\frac{2 \pi i}{\log q} \frac{k}{m\left(a_{\gamma}\right)}, k \in \mathbb{Z},
$$

for some facet $\gamma$ of $\Gamma(f)$ with perpendicular $a_{\gamma}$, and $m\left(a_{\gamma}\right) \neq 0$ (see [26, Theorem A, and Lemma 4.4]). Furthermore, if $\chi \neq \chi_{\text {triv }}$ and the order of $\chi$ does not divide any $m\left(a_{\gamma}\right) \neq 0$, where $\gamma$ is a facet of $\Gamma(f)$, then $Z(s, f, \chi)$ is a polynomial in $q^{-s}$, and its degree is bounded by a constant independent of $\chi$ (see $\left[26\right.$, Theorem B]). These two results imply that for $|z|_{K}$ big enough $E(z, f)$ is a finite $\mathbb{C}$-linear combination of functions of the form

$$
\chi(\operatorname{ac}(z))|z|_{K}^{\lambda}\left(\log _{q}\left(|z|_{K}\right)\right)^{\gamma},
$$

with coefficients independent of $z$, and with $\lambda \in \mathbb{C}$ a pole of

$$
\left(1-q^{-1-s}\right) Z\left(s, f, \chi_{\text {triv }}\right) \text { or of } Z(s, f, \chi), \chi \neq \chi_{\text {triv }},
$$

and $\gamma \in \mathbb{N}, \gamma \leqq$ (multiplicity of pole $\lambda$ ) -1 (see [2, Corollary 1.4.5]). Moreover all poles $\lambda$ appear effectively in this linear combination. Therefore

$$
|E(z, f)| \leqq c|z|_{K}^{-\beta_{f}+\epsilon}
$$

with $\epsilon>0$, and

$$
\beta_{f}:=\min _{\tau}\left\{\frac{\sigma\left(a_{\tau}\right)}{m\left(a_{\tau}\right)}\right\},
$$

where $\tau$ runs through all facets of $\Gamma(f)$ satisfying $m\left(a_{\tau}\right) \neq 0$. The point

$$
T_{0}=\left(\beta_{f}^{-1}, \ldots, \beta_{f}^{-1}\right) \in \mathbb{Q}^{m}
$$

is the intersection point of the boundary of the Newton polyhedron $\Gamma(f)$ with the diagonal $\Delta=\{(t, \ldots, t) \mid t \in \mathbb{R}\} \subset \mathbb{R}^{m}$. By combining estimation (11) and Theorem 1, we obtain the following result.

Theorem 2. Let $f(x) \in K[x]$ be non-degenerate with respect to its Newton polyhedron $\Gamma(f)$. Let $B \subset K^{m}$ a compact open subset. Then

$$
\left|E_{B}(z, f)\right| \leqq c|z|_{K}^{-\beta_{f}+\epsilon},
$$

for any $\epsilon>0$. 
We have to mention that the previous result is known by the experts, however the author did not find a suitable reference for the purposes of this article. If $K$ has characteristic $p>0$, the previous result is valid if $p$ does not divide the $m\left(a_{\tau}\right) \neq 0[26$, Corollary 6.1$]$.

\section{§3.2. Exponential Sums Associated with Quasi-homogeneous Polynomials}

Definition 2. Let $f(x) \in K[x], x=\left(x_{1}, \ldots, x_{m}\right)$ be a non-constant polynomial satisfying $f(0)=0$. The polynomial $f(x)$ is called quasi-homogeneous of degree $d$ with respect $\alpha=\left(\alpha_{1}, \ldots, \alpha_{m}\right) \in(\mathbb{N} \backslash\{0\})^{m}$, if it satisfies

$$
f\left(\lambda^{\alpha_{1}} x_{1}, \ldots, \lambda^{\alpha_{m}} x_{m}\right)=\lambda^{d} f(x), \text { for every } \lambda \in K .
$$

In addition, if $C_{f}(K)$ is the origin of $K^{m}$, then $f(x)$ is called a non-degenerate quasi-homogeneous polynomial.

The non-degenerate quasi-homogeneous polynomials are a subset of the non-degenerate polynomials with respect to the Newton polyhedron. For these type of polynomials the bound (11) can be improved:

$$
|E(z, f)| \leq c|z|_{K}^{-\beta_{f}}
$$

where $\beta_{f}=\frac{1}{d} \sum_{i=1}^{m} \alpha_{i}$. By using the techniques exposed in [25, Theorem 3.5], and [26, Lemma 2.4] follow that the poles of $\left(1-q^{-1-s}\right) Z\left(s, f, \chi_{\text {triv }}\right)$ and $Z(s, f, \chi), \chi \neq \chi_{\text {triv }}$, have the form

$$
s=-\frac{\sigma(\alpha)}{d}+\frac{2 \pi i}{\log q} \frac{k}{d}, k \in \mathbb{Z} .
$$

Then by using the same reasoning as before, we obtain (12). This estimate and Theorem 1 imply the following result.

Theorem 3. Let $f(x) \in K[x], x=\left(x_{1}, \ldots, x_{m}\right)$ be a non-degenerate quasi-homogeneous polynomial of degree $d$ with respect to $\alpha=\left(\alpha_{1}, \ldots, \alpha_{m}\right)$. Let $B \subset K^{m}$ be a compact open set. Then

$$
\left|E_{B}(z, f)\right| \leq c|z|_{K}^{-\beta_{f}} .
$$

If $K$ has characteristic $p>0$, the above result is valid, if $p$ does not divide $\sigma(\alpha)$. 


\section{$\S 4$. Fourier Transform of Measures Supported on Hypersurfaces}

Let $Y$ be a closed smooth submanifold of $K^{n}$ of dimension $n-1$. If

$$
I=\left\{i_{1}, \ldots, i_{n-1}\right\} \text { with } i_{1}<i_{2}<\cdots<i_{n-1}
$$

is a subset of $\{1, \ldots, n\}$ we denote by $\omega_{Y_{I}}$ the differential form induced on $Y$ by $d x_{i_{1}} \wedge d x_{i_{2}} \wedge \cdots \wedge d x_{i_{n-1}}$, and by $d \sigma_{Y_{I}}$ the corresponding measure on $Y$. The canonical measure of $Y$ is defined as

$$
d \sigma_{Y}=\sup _{I}\left\{d \sigma_{Y_{I}}\right\}
$$

where $I$ runs through all the subsets of form (13). Given $S$ a compact open subset of $K^{n}$ with characteristic function $\Theta_{S}$, we define $d \mu_{Y, S}=d \mu_{Y}=\Theta_{S} d \sigma_{Y}$. The canonical measure $d \mu_{Y}$ was introduced by Serre in [14]. The Fourier transform of $d \mu_{Y}$ is defined as

$$
\widehat{d \mu_{Y}(\xi)}=\int_{Y} \Psi(-[x, \xi]) d \mu_{Y}(x),
$$

where $[x, y]:=\sum_{i=1}^{n} x_{i} y_{i}$, with $x, y \in K^{n}$. The analysis of the decay of $\left|\widehat{d \mu_{Y}(\xi)}\right|$ as $\|\xi\|_{K}:=\max _{i}\left\{\left|\xi_{i}\right|_{K}\right\}$ approaches infinity plays a central role in this paper. This analysis can be simplified taking into account the following facts. Any compact open set of $K^{n}$ is a finite union of classes modulo $\pi^{e}$, by taking $e$ big enough, and taking into account that $Y \cap y+\left(\pi^{e} R_{K}\right)^{n}$ is a hypersurface of the form

$$
\left\{x \in y+\left(\pi^{e} R_{K}\right)^{n} \mid x_{n}=\phi\left(x_{1}, \ldots, x_{n-1}\right)\right\}
$$

with $\phi$ an analytic function satisfying

$$
\phi(0)=\frac{\partial \phi}{\partial x_{1}}(0)=\cdots=\frac{\partial \phi}{\partial x_{n-1}}(0)=0
$$

(see [14, p. 147]), we may assume that $Y$ is a hypersurface of the form $x_{n}-$ $\phi\left(x_{1}, \ldots, x_{n-1}\right)=0$, with $\phi$ satisfying (14). In this case $d \sigma_{Y}(x)=\left|d x_{1}\right| \ldots$ $\left|d x_{n-1}\right|$, the normalized Haar measure of $K^{n-1}$.

Finally we want to mention that if $X=\left\{x \in K^{n} \mid f(x)=0\right\}$ is a hypersurface then

$$
\frac{d x_{1} \ldots d x_{n-1}}{\left|\frac{\partial f}{\partial x_{n}}\right|_{K}}
$$


is a measure on a neighborhood of $X$ provided that $\left|\frac{\partial f}{\partial x_{n}}\right|_{K} \neq 0$ (see [7, Section 7.6]). This measure is not intrinsic to $X$, but if $S$ is small enough, it coincides with $d \mu_{X}=\Theta_{S} d \sigma_{X}$ for a polynomial of type $f(x)=x_{n}-\phi\left(x_{1}, \ldots, x_{n-1}\right)$. The Serre measure allow us to define $\widehat{d \mu_{Y}(\xi)}$ intrinsically for an arbitrary submanifold $Y$.

The rest of this section is dedicated to describe the asymptotics of $\widehat{d \mu_{Y}}$ when $\phi$ is a non-degenerate polynomial with respect $\Gamma(\phi)$.

Theorem 4. Let $\phi(x) \in R_{K}[x], x=\left(x_{1}, \ldots, x_{n-1}\right)$, be a non-degenerate polynomial with respect to its Newton polyhedron $\Gamma(\phi)$. Let $\Theta_{S}$ be the characteristic function of a compact open set $S$, let

$$
Y=\left\{x \in K^{n} \mid x_{n}=\phi\left(x_{1}, \ldots, x_{n-1}\right)\right\},
$$

and let $d \mu_{Y}=\Theta_{S} d \sigma_{Y}$. Then

$$
\left|\widehat{d \mu_{Y}(\xi)}\right| \leq c\|\xi\|_{K}^{-\beta},
$$

for $0 \leq \beta \leq \beta_{\phi}-\epsilon$, for $\epsilon>0$. Furthermore, if $\phi$ is a non-degenerate quasihomogeneous polynomial, (15) is valid for $0 \leq \beta \leq \beta_{\phi}$.

Proof. Since $S$ is compact by passing to a sufficiently fine covering

$$
\bigcup_{i}\left(x_{i}, \phi\left(x_{i}\right)\right)+\left(\pi^{e_{0}} R_{K}\right)^{n}
$$

with $e_{0}>0$, we may suppose that $S=\left(x_{i}, \phi\left(x_{i}\right)\right)+\left(\pi^{e_{0}} R_{K}\right)^{n}$. In the case $x_{i}=0$,

$$
\widehat{d \mu_{Y}(\xi)}=\int_{\left(\pi^{e} 0 R_{K}\right)^{n-1}} \Psi\left(-\xi_{n} \phi(x)-\left[x, \xi^{\prime}\right]\right)|d x|,
$$

where $\xi^{\prime}=\left(\xi_{1}, \ldots, \xi_{n-1}\right)$. If $\xi^{\prime}=0$, Theorem 2 implies that

$$
\left|\widehat{d \mu_{Y}(\xi)}\right| \leq c\left|\xi_{n}\right|_{K}^{-\beta}=c\|\xi\|_{K}^{-\beta},
$$

for $0 \leq \beta \leq \beta_{\phi}-\epsilon, \epsilon>0$. Furthermore, if $\phi$ is a non-degenerate quasihomogeneous polynomial then (17) is valid for $0 \leq \beta \leq \beta_{\phi}$ (cf. Theorem $3)$.

Since $\left.\widehat{d \mu_{Y}(\xi)}=d \mu_{Y} \widehat{\left(\xi_{n}\right.}, \xi^{\prime}\right)$ is a continuous function with respect to $\xi^{\prime}$, estimation (17) remains valid if

$$
\frac{\left|\xi_{i}\right|_{K}}{\left|\xi_{n}\right|_{K}} \leq c, i=1, \ldots, n-1,
$$


for some small positive constant $c$. Then we may suppose that

$$
\frac{\left|\xi_{i}\right|_{K}}{\left|\xi_{n}\right|_{K}}>c, i=1, \ldots, n-1 .
$$

Since $\left(\pi^{e_{0}} R_{K}\right)^{n-1}$ is small enough, (19) implies that the system of equations

$$
\frac{\partial \phi(x)}{\partial x_{j}}=\frac{\xi_{i}}{\xi_{n}}, j=1, \ldots, n-1,
$$

does not have solutions in $\left(\pi^{e_{0}} R_{K}\right)^{n-1}$, and then the critical set of the polynomial

$$
F(x, \xi)=\xi_{n} \phi(x)+\left[x, \xi^{\prime}\right]
$$

does not meet $\left(\pi^{e_{0}} R_{K}\right)^{n-1}$ if $\xi_{n} \neq 0$. By applying Theorem 1 , it follows that

$$
\widehat{d \mu_{Y}(\xi)}=0 \text {, for }\|\xi\|_{K} \text { big enough. }
$$

Then for $\|\xi\|_{K}$ big enough, (17) and (20) imply that

$$
\left|\widehat{d \mu_{Y}(\xi)}\right| \leq A\|\xi\|_{K}^{-\beta}, \text { for } 0 \leq \beta \leq \beta_{\phi} .
$$

In the case $x_{i} \neq 0$, by using the fact that the origin is the only critical point of $\phi$, a similar reasoning shows that $\widehat{d \mu_{Y}(\xi)}=0$, for $\|\xi\|_{K}$ big enough. Therefore estimation (21) is valid for any compact open set $S$.

\section{§4.1. Restriction of the Fourier Transform to Non-degenerate Hypersurfaces}

Let $X$ be a submanifold of $K^{n}$ with $d \sigma_{X}$ its canonical measure. We set $d \mu_{Y, S}=\Theta_{S} d \sigma_{Y}$, where $\Theta_{S}$ is the characteristic function of a compact open set $S$ in $K^{n}$. We say that the $L^{\rho}$ restriction property is valid for $X$ if there exists a $\tau(\rho)$ so that

$$
\left(\int_{X}|\mathcal{F} g(\xi)|_{K}^{\tau} d \mu_{X, S}(\xi)\right)^{\frac{1}{\tau}} \leq c_{\tau, \rho}(S)\|g\|_{L^{\rho}}
$$

holds for each $g \in \mathbb{S}\left(K^{n}\right)$ and any compact open set $S$ of $K^{n}$

The restriction problem in $\mathbb{R}^{n}$ (see e.g. [17, Chapter VIII]) was first posed and partially solved by Stein [5]. This problem have been intensively studied during the last thirty years [1], [17], [19], [24]. Recently Mockenhaupt and 
Tao have studied the restriction problem in $\mathbb{F}_{q}^{n}[12]$. In this paper we study the restriction problem in the non-archimedean field setting. More precisely, in the case in which $X$ is a non-degenerate hypersurface and $\tau=2$. The proof of the restriction property in the non-archimedean case uses the Lemma of interpolation of operators (see e.g. [17, Chapter IX]) and the estimates for oscillatory integrals obtained in the previous section. The interpolation Lemma given in $[17$, Chapter IX] is valid in the non-archimedean case. For the sake of completeness we rewrite this lemma here.

Let $\left\{U^{z}\right\}$ be a family of operators on the strip $a \leq \operatorname{Re}(z) \leq b$ defined by

$$
\left(U^{z} g\right)(x)=\int_{K^{n}} \mathfrak{K}_{z}(x, y) g(y)|d y|,
$$

where the kernels $\mathfrak{K}_{z}(x, y)$ have a fixed compact support and are uniformly bounded for $(x, y) \in K^{n} \times K^{n}$ and $a \leq \operatorname{Re}(z) \leq b$. We also assume that for each $(x, y)$, the function $\mathfrak{K}_{z}(x, y)$ is analytic in $a<\operatorname{Re}(z)<b$ and is continuous in the closure $a \leq \operatorname{Re}(z) \leq b$, and that

$$
\left\{\begin{array}{l}
\left\|U^{z} g\right\|_{L^{\tau_{0}}} \leq M_{0}\|g\|_{L^{\rho_{0}}}, \text { when } \operatorname{Re}(z)=a, \\
\left\|U^{z} g\right\|_{L^{\tau_{1}}} \leq M_{1}\|g\|_{L^{\rho_{1}}}, \text { when } \operatorname{Re}(z)=b ;
\end{array}\right.
$$

here $\left(\tau_{i}, \rho_{i}\right)$ are two pairs of given exponents with $1 \leq \tau_{i}, \rho_{i} \leq \infty$.

Lemma 2 (Interpolation Lemma [17, Chapter IX]). Under the above hypotheses,

$$
\left\|U^{a(1-\theta)+b \theta} g\right\|_{L^{\tau}} \leq M_{0}^{1-\theta} M_{1}^{\theta}\|g\|_{L^{\rho}}
$$

where $0 \leq \theta \leq 1, \frac{1}{\tau}=\frac{(1-\theta)}{\tau_{0}}+\frac{\theta}{\tau_{1}}$, and $\frac{1}{\rho}=\frac{(1-\theta)}{\rho_{0}}+\frac{\theta}{\rho_{1}}$.

Theorem 5. Let $\phi(x) \in K[x], x=\left(x_{1}, \ldots, x_{n-1}\right)$, be a non-degenerate polynomial with respect to its Newton polyhedron $\Gamma(\phi)$. Let

$$
Y=\left\{x \in K^{n} \mid x_{n}=\phi\left(x_{1}, \ldots, x_{n-1}\right)\right\}
$$

with the measure $d \mu_{Y, S}=\Theta_{S} d \sigma_{Y}$, where $\Theta_{S}$ is the characteristic function of a compact open subset $S$ of $K^{n}$. Then

$$
\left(\int_{Y}|\mathcal{F} g(\xi)|_{K}^{2} d \mu_{Y}(\xi)\right)^{\frac{1}{2}} \leq c(Y)\|g\|_{L^{\rho}},
$$


holds for each $1 \leq \rho<\frac{2\left(1+\beta_{\phi}\right)}{2+\beta_{\phi}}$. Furthermore, if $\phi$ is a non-degenerate quasihomogeneous polynomial, (22) holds for each $1 \leq \rho \leq \frac{2\left(1+\beta_{\phi}\right)}{2+\beta_{\phi}}$.

Proof. We first note that

$$
\begin{aligned}
\int_{Y}|\mathcal{F} g(\xi)|_{K}^{2} d \mu_{Y, S}(\xi) & =\int_{Y} \mathcal{F} g(\xi) \overline{\mathcal{F} g(\xi)} d \mu_{Y, S}(\xi) \\
& =\int_{K^{n}}(T g)(x) \overline{g(x)}|d x|
\end{aligned}
$$

where $(T g)(x)=(g * \mathfrak{K})(x)$ with

$$
\mathfrak{K}(x)=\int_{Y} \Psi([x, \xi]) d \mu_{Y, S}(\xi)=d \widehat{\mu_{Y, S}(-x)} .
$$

The theorem follows from (23) by Hölder's inequality if we show that

$$
\|T(g)\|_{L^{\rho_{0}^{\prime}}} \leq c\|g\|_{L^{\rho_{0}}}
$$

where $\rho_{0}^{\prime}$ is the dual exponent of $\rho_{0}$. Now we define $\mathfrak{K}_{z}(x)$ as equal to

$$
\gamma(z) \int_{K^{n}} \Psi([x, \xi])\left|\xi_{n}-\phi\left(\xi^{\prime}\right)\right|_{K}^{-1+z} \eta\left(\xi_{n}-\phi\left(\xi^{\prime}\right)\right) \Theta_{S}\left(\xi^{\prime}, \phi\left(\xi^{\prime}\right)\right)|d \xi|,
$$

where $\gamma(z)=\left(\frac{1-q^{-z}}{1-q^{-1}}\right), \xi^{\prime}=\left(\xi_{1}, \ldots, \xi_{n-1}\right), \eta(\xi)$ is the characteristic function of the ball $P_{K}^{e_{0}}, e_{0} \geq 1$, and $\operatorname{Re}(z)>0$. By making $y=\xi_{n}-\phi\left(\xi^{\prime}\right)$ in the above integral we obtain

$$
\mathfrak{K}_{z}(x)=\zeta_{z}\left(x_{n}\right) \mathfrak{K}(x)
$$

with

$$
\zeta_{z}\left(x_{n}\right)=\gamma(z) \int_{K} \Psi\left(x_{n} y\right)|y|_{K}^{-1+z} \eta(y)|d y|, \operatorname{Re}(z)>0 .
$$

On the other hand,

$$
\zeta_{z}\left(x_{n}\right)=\left\{\begin{array}{cll}
q^{-e_{0} z}, & \text { if } & \left|x_{n}\right|_{K} \leq q^{e_{0}} \\
\left(\frac{1-q^{z-1}}{1-q^{-1}}\right)\left|x_{n}\right|_{K}^{-z}, & \text { if } & \left|x_{n}\right|_{K}>q^{e_{0}},
\end{array}\right.
$$

(for a similar calculation the reader can see [20, page 54]), then $\zeta_{z}\left(x_{n}\right)$ has an analytic continuation to the complex plane as an entire function; also $\zeta_{0}\left(x_{n}\right)=$ 1 , and $\left|\zeta_{z}\left(x_{n}\right)\right| \leq c\left|x_{n}\right|_{K}^{-\operatorname{Re}(z)}$ where $\left|x_{n}\right|_{K} \geq q^{e_{0}}$. Therefore $\zeta_{z}\left(x_{n}\right)$ has an analytic continuation to an entire function satisfying the following properties: 
(i) $\mathfrak{K}_{0}(x)=\mathfrak{K}(x)$,

(ii) $\left|\mathfrak{K}_{-\beta+i \gamma}(x)\right| \leq c$, for $x \in K^{n}, \gamma \in \mathbb{R}$, and $0 \leq \beta \leq \beta_{\phi}-\epsilon, \epsilon>0$,

(iii) $\left|\mathcal{F} \mathfrak{K}_{1+i \gamma}(\xi)\right| \leq c$, for $\xi \in K^{n}$, and $\gamma \in \mathbb{R}$.

In fact (ii) follows from Theorem 4 , and (iii) is an immediate consequence of the definition of $\mathfrak{K}_{z}(x)$.

Now we consider the analytic family of operators $T_{z}(g)=\left(g * \mathfrak{K}_{z}\right)(x)$. From (ii) one has

$$
\left\|T_{-\beta+i \gamma}(g)\right\|_{L^{\infty}} \leq c\|g\|_{L^{1}}
$$

for $0 \leq \beta \leq \beta_{\phi}-\epsilon, \epsilon>0$, and $\gamma \in \mathbb{R}$, and from (iii) and Plancherel's Theorem one gets

$$
\left\|T_{1+i \gamma}(g)\right\|_{L^{2}} \leq c\|g\|_{L^{2}}
$$

for $\gamma \in \mathbb{R}$. By applying the Interpolation Lemma with

$$
\theta=\frac{\beta}{1+\beta},
$$

we obtain

$$
\left\|T_{0}(g)\right\|_{L^{\rho^{\prime}}} \leq c\|g\|_{L^{\rho}},
$$

with $\rho^{\prime}$ the dual exponent of $\rho=\frac{2(1+\beta)}{2+\beta}$, and $0 \leq \beta \leq \beta_{\phi}-\epsilon, \epsilon>0$. Therefore the previous estimate for $\left\|T_{0}(g)\right\|_{L^{\rho^{\prime}}}$ is valid for $1 \leq \rho \leq \frac{2\left(1+\beta_{\phi}-\epsilon\right)}{2+\beta_{\phi}-\epsilon}$. In the quasi-homogeneous case the estimate is valid for $1 \leq \rho \leq \frac{2\left(1+\beta_{\phi}\right)}{2+\beta_{\phi}}$.

Our proof of Theorem 5 is strongly influenced by Stein's proof for the restriction problem in the case of a smooth hypersurface in $\mathbb{R}^{n}$ with non-zero Gaussian curvature [16].

\section{$\S 5 . \quad$ Asymptotic Decay of Solutions of Wave-type Equations}

Like in the classical case [19], the decay of the solutions of wave-type pseudo-differential equations can be deduced from the restriction theorem proved in the previous section, taking into account that the following two problems are completely equivalent if $\frac{1}{\rho}+\frac{1}{\sigma}=1$ : 
Problem 1. For which values of $\rho, 1 \leq \rho<2$, is it true that $f \in$ $L^{\rho}\left(K^{n}\right)$ implies that $\mathcal{F} f$ has a well-defined restriction to $Y$ in $L^{2}\left(d \mu_{Y, S}\right)$ with

$$
\left(\int_{Y}|\mathcal{F} f|^{2} d \mu_{Y, S}\right)^{\frac{1}{2}} \leq c_{\rho}\|f\|_{L^{\rho}} ?
$$

Problem 2. For which values of $\sigma, 2<\sigma \leq \infty$, is it true that the distribution $g d \mu_{Y, S}$ for each $g \in L^{2}\left(d \mu_{Y, S}\right)$ has Fourier transform in $L^{\sigma}\left(K^{n}\right)$ with

$$
\left\|\mathcal{F}\left(g d \mu_{Y, S}\right)\right\|_{L^{\sigma}} \leq c_{\sigma}\left(\int_{Y}|g|^{2} d \mu_{Y, S}\right)^{\frac{1}{2}} ?
$$

\section{§5.1. Wave-type Equations with Non-degenerate Symbols}

Theorem 6 (Main Result). Let $\phi(\xi) \in K[\xi], \xi=\left(\xi_{1}, \ldots, \xi_{n}\right)$ be a non-degenerate polynomial with respect $\Gamma(\phi)$. Let

$$
\begin{aligned}
H: \mathbb{S}\left(K^{n}\right) & \longrightarrow \\
\Phi & \longrightarrow \mathcal{F}_{(\tau, \xi) \longrightarrow(x, t)}^{-1}\left(|\tau-\phi(\xi)|_{K} \mathcal{F}_{(x, t) \longrightarrow(\tau, \xi)} \Phi\right),
\end{aligned}
$$

be a pseudo-differential operator with symbol $|\tau-\phi(\xi)|_{K}$. Let $u(x, t)$ be the solution of the following initial value problem:

$$
\left\{\begin{array}{l}
(H u)(x, t)=0, x \in K^{n}, t \in K, \\
u(x, 0)=f_{0}(x),
\end{array}\right.
$$

where $f_{0}(x) \in \mathbb{S}\left(K^{n}\right)$, then

$$
\|u(x, t)\|_{L^{\sigma}\left(K^{n+1}\right)} \leq A\left\|f_{0}(x)\right\|_{L^{2}\left(K^{n}\right)},
$$

for $\frac{2\left(1+\beta_{\phi}\right)}{\beta_{\phi}}<\sigma \leq \infty$. Furthermore, if $\phi$ is a quasi-homogeneous polynomial, (24) is valid for $\frac{2\left(1+\beta_{\phi}\right)}{\beta_{\phi}} \leq \sigma \leq \infty$.

Proof. Since

$$
\begin{aligned}
u(x, t) & =\int_{K^{n}} \Psi(t \phi(\xi)+[x, \xi]) \mathcal{F} f_{0}(\xi)|d \xi| \\
& =\int_{Y} \Psi([\underline{x}, \underline{\xi}]) \mathcal{F} f_{0}(\underline{\xi}) d \mu_{Y, S} \underline{(\xi)},
\end{aligned}
$$


where $\underline{\xi}=\left(\xi, \xi_{n+1}\right) \in K^{n+1}, \underline{x}=(x, t) \in K^{n+1}$,

$$
Y=\left\{\underline{\xi} \in K^{n+1} \mid \xi_{n+1}=\phi(\xi)\right\}
$$

and $d \mu_{Y, S}=\Theta_{S} d \sigma_{Y}$, with $\Theta_{S}$ the characteristic function of a compact open set $S$ containing the support of $\mathcal{F} f_{0}$. By applying Theorem 5 , replacing $n$ with $n+1$, and dualizing, one gets

$$
\|u(x, t)\|_{L^{\sigma}\left(K^{n+1}\right)} \leq A\left\|f_{0}(x)\right\|_{L^{2}\left(K^{n}\right)},
$$

where $\sigma=\frac{2(1+\beta)}{\beta}$ is the dual exponent of $\rho$ in Theorem 5 , and $0 \leq \beta<\beta_{\phi}$, therefore $(25)$ is valid for $\frac{2\left(1+\beta_{\phi}\right)}{\beta_{\phi}}<\sigma \leq \infty$.

The following corollary follows immediately from the previous theorem by using the fact that $\mathbb{S}\left(K^{n}\right)$ is dense in $L^{\sigma}\left(K^{n}\right)$ for $1 \leq \sigma<\infty$.

Corollary 1. With the hypothesis of Theorem 6 , if $f_{0} \in L^{2}\left(K^{n}\right)$, then $u(x, t) \in L^{\sigma}\left(K^{n+1}\right)$, for $\frac{2\left(1+\beta_{\phi}\right)}{\beta_{\phi}}<\sigma<\infty$. Furthermore, if $\phi$ is a quasihomogeneous polynomial, $u(x, t) \in L^{\sigma}\left(K^{n+1}\right)$, for $\frac{2\left(1+\beta_{\phi}\right)}{\beta_{\phi}} \leq \sigma<\infty$.

\section{§5.2. Wave-type Equations with Quasi-homogeneous Symbols}

As a consequence of the previous theorem we obtain the following two corollaries.

Corollary 2. With the hypothesis of Theorem 6 , if $\phi(\xi)=\xi_{1}^{2}+\cdots+\xi_{n}^{2}$, then

$$
\|u(x, t)\|_{L^{\frac{2(2+n)}{n}}\left(K^{n+1}\right)} \leq A\left\|f_{0}(x)\right\|_{L^{2}\left(K^{n}\right)} .
$$

Corollary 3. With the hypothesis of Theorem 6 , if $\phi(\xi)=\xi^{d}$, then

$$
\|u(x, t)\|_{L^{2(d+1)}\left(K^{2}\right)} \leq A\left\|f_{0}(x)\right\|_{L^{2}(K)} .
$$

In particular if $d=3$, then

$$
\|u(x, t)\|_{L^{8}\left(K^{2}\right)} \leq A\left\|f_{0}(x)\right\|_{L^{2}(K)} .
$$




\section{References}

[1] Bourgain, J., "Some new estimates on oscillatory integrals" in Essays on Fourier Analysis in Honor of Elias M. Stein (Princeton, 1991), Math. Ser. 42, Princeton Univ. Press, Princeton, 1995, 83-112.

[2] Denef, J., Report on Igusa's Local Zeta Function, Séminaire Bourbaki 43 (1990-1991), exp. 741; Astérisque 201-202-203 (1991), 359-386. Available at http://www.wis.kuleuven.ac.be/algebra/denef.html.

[3] 13(3) (1995), 289-295.

[4] Denef, J. and Hoornaert, K., Newton polyhedra and Igusa local zeta function, J. Number Theory, 89(1) (2001), 31-64.

[5] Fefferman, C., Inequalities for strongly singular convolution operators, Acta. Math., 124 (1970), 9-36.

[6] Gelfand, I. M., Graev, M. I. and Piatetski-Shapiro, I. I., Representation theory and automorphic functions, Saunders, Philadelphia, 1969.

[7] Igusa, J.-I., An introduction to the theory of local zeta functions, AMS /IP Stud. Adv. Math., 14, 2000.

[8] Kochubei, A. N., Pseudodifferential equations and stochastics over non-archimedean fields, Marcel Dekker, 2001.

[9] , A Schrödinger type equation over the field of $p$-adic numbers, J. Math. Phys., 34 (1993), 3420-3428.

[10] Kenig, Carlos, Ponce, Gustavo and Vega, Luis, Oscillatory integrals and regularity of dispersive equations, Indiana Univ. Math. J., 40(1) (1991), 33-69.

[11] Khrennikov, A., p-Adic Valued Distributions in Mathematical Physics, Kluwer, Dordrecht, 1994

[12] Mockenhaupt, Gerd and Tao, Terence, Restriction and Kakeya phenomena for finite fields, Duke Math. J., 121 (2004), 35-74.

[13] Ruelle, Ph., Thiran, E., Verstegen, D. and Weyers, J., Quantum mechanics on $p$-adic fields, J. Math. Phys., 30 (1989), (12), 2854-2874.

[14] Serre, J.-P., Quelques applications du théor ème de densité de Chebotarev, (French) [Some applications of the Chebotarev density theorem] Inst. Hautes Études Sci. Publ. Math., 54 (1981), 323-401.

[15] Stein, E. M., "Some problems in harmonic analysis" in Harmonic Analysis in Euclidean Spaces, Part I (Williamstown, Mass., 1978), Proc. Sympos. Pure Math., 35 (1979), 3-20.

[16] _ "Oscillatory integrals in Fourier analysis" in Beijing Lectures in Harmonic Analysis (ed.). Ann. of Math. Stud. 112, Princeton Univ. Press, 1986.

[17] - Harmonic analysis: Real-Variable Methods, Orthogonality, and Oscillatory Integrals, Princeton Math. Ser., 43, Monogr. Harmon. Anal., 3, Princeton Univ. Press, Princeton, 1993.

[18] Stein, E. M. and Weiss, G., Introduction to Fourier analysis on Euclidean Spaces, Princeton Univ. Press, 1971.

[19] Strichartz, R. S., Restrictions of the Fourier transforms to quadratic surfaces and decay of solutions of wave equations, Duke Math. J., 44 (1977), 705-774.

[20] Vladimirov, V. S., Tables of Integrals of Complex-valued Functions of p-Adic arguments, http://www.arxiv.org/abs/math-ph/9911027.

[21] Vladimirov, V. S. and Volovich, I. V., p-Adic quantum mechanics, Comm. Math. Phys., 123 (1989), 659-676.

[22] Vladimirov, V. S., Volovich, I. V. and Zelenov, E. I., p-adic Analysis and mathematical physics, Series on Soviet and East European Mathematics, 1. World Scientific Publishing Co., Inc., River Edge, NJ, 1994.

[23] Taibleson, M. H., Fourier analysis on local fields, Princeton University Press, Princeton, N.J.; University of Tokyo Press, Tokyo, 1975.

[24] Tomas, P. A., A restriction theorem for the Fourier transform, Bull. Amer. Math. Soc., 81 (1975), 477-478. 
[25] Zuniga-Galindo, W. A., Igusa's local zeta functions of semiquasihomogeneous polynomials, Trans. Amer. Math. Soc., 353 (2001), 3193-3207.

[26] $\longleftarrow$, Local zeta functions and Newton polyhedra, Nagoya Math. J., 170 (2003), $31-58$. 